\begin{tabular}{|c|l|}
\hline Title & Low temperature transport in dual-gated SET s fabricated by selective area metal organic vapor phase epitaxy \\
\hline Author(s) & $\begin{array}{l}\text { Motohisa, J.; van der Wiel, W. G.; Elzerman, J. M.; De Franceschi, S.; Nakajima, F.; Ogasawara, Y.; Fukui, T.; } \\
\text { Kouwenhoven, L. P. }\end{array}$ \\
\hline Citation & $\begin{array}{l}\text { Physica E: Low-dimensional Systems and Nanostructures, 13(2-4), 687-690 } \\
\text { https://doi.org/10.1016/S1386-9477(02)00259-X }\end{array}$ \\
\hline Issue Date & 2002-04 \\
\hline Doc URL & http://hdl.handle.net/2115/5535 \\
\hline Type & article (author version) \\
\hline File Information & PE13(2-4).pdf \\
\hline
\end{tabular}

Instructions for use 


\title{
Low temperature transport in dual-gated SETs fabricated by selective area metalorganic vapor phase epitaxy
}

\author{
J. Motohisa ${ }^{\mathrm{a}, *}$, W.G. van der Wiel ${ }^{\mathrm{b}}$, J.M. Elzerman ${ }^{\mathrm{b}}$, \\ S. De Franceschi b, F. Nakajima ${ }^{\text {a }}$, Y. Ogasawara ${ }^{\text {a }}$, T. Fukui a, \\ L. P. Kouwenhoven ${ }^{b}$ \\ ${ }^{a}$ Research Center for Integrated Quantum Electronics, Hokkaido University, North \\ 13 West 8, Sapporo 060-8628, Japan \\ ${ }^{\mathrm{b}}$ Department of Applied Physics and DIMES, Delft University of Technology, PO \\ Box 5046, 2600 GA Delft, The Netherlands
}

\begin{abstract}
We describe transport measurements in a novel dual-gated single electron transistor (SET) based on a quantum dot (QD) fabricated by selective area (SA) growth of metalorganic vapor phase epitaxy (MOVPE). We observed, for the first time, clear Coulomb oscillations fabricated in combination with direct growth and lithographically defined metal gates, and achieved nearly independent control of the QD potential and the tunneling barrier height. We also were able to observe a signature of Kondo resonance when the coupling between leads and a quantum dot was sufficiently strong. (This article is published in Physica E vol.13, No. 2-4, pp.687-690 (2002).)
\end{abstract}

Key words: single electron transistor, selective-area metalorganic vapor phase epitaxy, Coulomb oscillation, Kondo effect

PACS: 73.23.Hk 73.63.Kv 81.15.Gh

\section{Introduction}

Single electron transport through semiconductor quantum dots (QDs) attracts considerable recent attention. From the

\footnotetext{
* Corresponding author.

Email address: motohisa@rciqe.hokudai.ac.jp (J. Motohisa).
}

application point of view, single electron devices utilizing Coulomb blockade effect have a potential to realize integrated circuits with high-density integration and low power-consumption[1]. From the physics point of view, spectroscopic study of discrete energy states of QDs analogous to atoms is carried out by using single electron transport[2]. Furthermore, if there is a net spin on a $\mathrm{QD}$, it can be consid- 
ered as localized magnetic impurities with tunable coupling between conduction electrons, which offers a playground of tunable Kondo physics[3]. In this case, very small quantum dots are required.

We have reported on the fabrication of single electron devices by using selective area metalorganic vapor phase epitaxy (SA-MOVPE) on partially masked substrates[4-6]. In particular, we have recently proposed and demonstrated[7] a structure having much stronger lateral confinement as compared to the conventional structures by utilizing the structure grown on zig-zag-shaped mask patterns. However, Coulomb oscillations were observed only near the pinch-off region of the single electron transistor (SET). This is because the gate wrapped around both the QD and adjacent regions, and the gate changes both QD potential and the height of tunneling barriers, and it is a major drawback of such epitaxially grown structures with simple gate geometry[4-8]. In this study, we propose and fabricate a device which has two gates, namely, a side gate and a top gate, to overcome such shortcomings. We observe clear Coulomb oscillations in a wide range of gate voltages. A signature of the Kondo effect is also observed when the coupling between QD and leads is sufficiently strong.

\section{Sample Structure and Experi- ment}

The SEM image of our quantum dot device and its schematic are shown in Figs. 1(a) and $1(\mathrm{~b})$, respectively. The structure is formed by SA-MOVPE on a masked substrate with zig-zag shaped opening regions, and successive electron beam lithography and lift-off processes for the gate electrodes. This approach for the formation of nanostructures enables us to form extremely small QDs, going beyond the limit of current lithographic techniques[4,8]. Details of the growth process and fabrication are reported elsewhere[7]. By applying a negative top gate voltage, a quantum dot is formed in between the constriction, as shown in Fig.1(b)[7]. The top gate is also used to control the height of tunnel barriers between the QD and the leads formed by quantum wires. The geometrical diameter of $\mathrm{QD}$ in the present sample is estimated to be $320 \mathrm{~nm}$, and is reduced by the application of a negative top gate voltage. In contrast, the side gate mainly changes the potential of the QD. Therefore, dual-gated structure gives us a mean to control the electrostatic potential of QD independently, retaining strong lateral confinement and minimizing the change of dot size and tunneling barrier height. The measurements are performed in a dilution refrigerator with a base temperature of $15 \mathrm{mK}$ and conductance through the QD is measured with a constant source-drain DC bias, $V_{D S}$, of $10 \mu \mathrm{V}$. The differential conductance, $d I_{D S} / d V_{D S}$, is measured using a lock-in technique with an excitation AC voltage of $5 \mu \mathrm{V}$ and frequency of $17.77 \mathrm{~Hz}$. DC voltage $V_{D S}$ is simultaneously applied to measure the $d I_{D S} / d V_{D S}$ at finite bias voltage.

\section{$3 \quad$ Results and Discussions}

Without applying a negative top gate voltage, $V_{T G}$, the conductance through the QD hardly changes when sweeping the side gate voltage, $V_{S G}$. However, by applying a voltage more negative than about $-250 \mathrm{mV}$ to $V_{T G}$, the conductance shows 


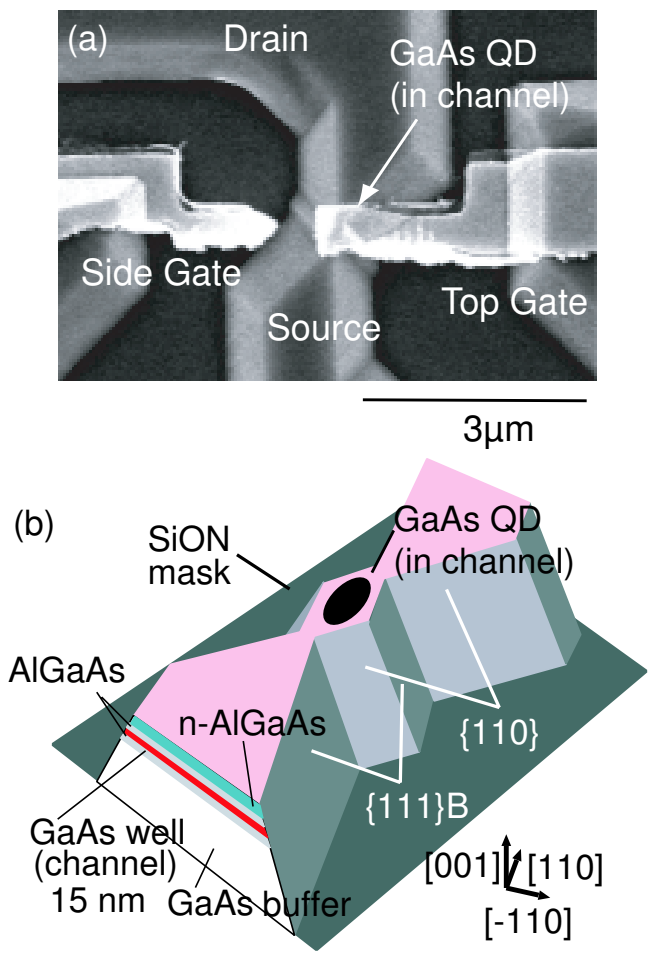

Fig. 1. (a) SEM image of the device and (b) its schematic illustration.

Coulomb oscillations as a function of $V_{S G}$. Figure 2 shows typical Coulomb oscillations for different top gate voltages. The Coulomb peaks shift to more negative side gate values when the top gate bias is made more positive. These results clearly indicate that a QD is formed in our device by applying a sufficiently negative voltage to the top gate.

Figure 3 shows a grey-scale plot of the differential conductance, $d I_{D S} / d V_{D S}$, for $V_{T G}=-299 \mathrm{mV}$, plotted as a function of both $V_{D S}$ and $V_{S G}$. We observe Coulomb diamonds. The asymmetry of the diamonds around $V_{D S}=0$ indicates that the tunneling capacitances of two tunneling junctions are not identical. This is probably because of the shape around the dot and tunneling barriers are not completely symmetric. In addition, the Coulomb gap $U_{\mathrm{C}}$ strongly depends on the side gate voltage $V_{S G}$. The obtained maximum and

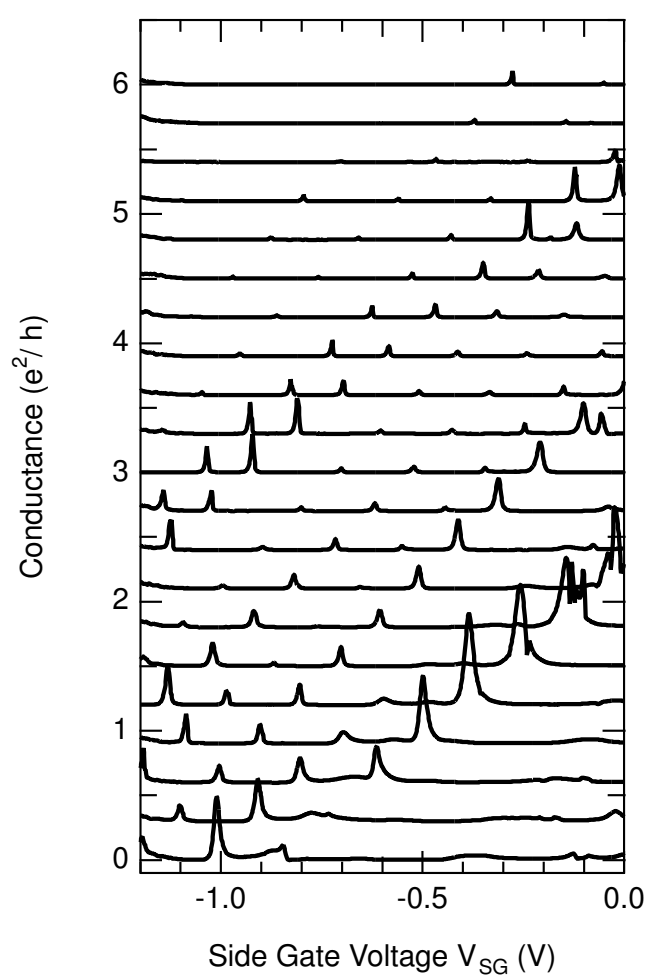

Fig. 2. Linear conductance of the device measured at base temperature $(\sim 15 \mathrm{mK})$ as a function of side gate voltage $V_{S G}$ for fixed top gate voltage $V_{T G}$. Top gate voltage $V_{T G}$ is varied from $-305 \mathrm{mV}$ (top) to $-285 \mathrm{mV}$ (bottom) with $1 \mathrm{mV}$ step size.

minimum values for $U_{\mathrm{C}}$ are $3.30 \mathrm{mV}$ and $1.34 \mathrm{mV}$, respectively. A rough estimate of the dot diameter from these results gives a value smaller than the geometrical size of $320 \mathrm{~nm}$. It is noted that $U_{\mathrm{C}}$ does not change monotonically as $V_{\mathrm{SG}}$. Therefore, the change of the dot size by $V_{S G}$ is not the only reason for such dependence and is not fully clarified yet. The magnetic field dependence of the Coulomb oscillation (not shown here) strongly suggests that the number of electrons in QD is very small and that the carrier confinement is strong. The $B$-dependence suggests similarities with the shell structure of artificial atoms as observed in vertical quantum dots[2].

The results obtained so far correspond to the regime where the tunneling resistances 


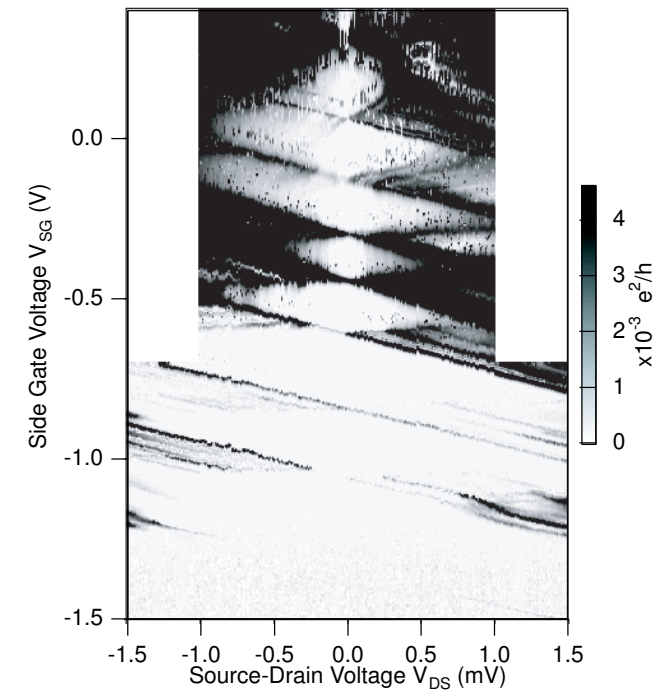

Fig. 3. Grey scale plot of the differential conductance $d I_{D S} / d V_{D S}$, plotted as a function of both $V_{S D}$ and $V_{S G}$. The top gate voltage is $-299 \mathrm{mV}$.

of the barriers are high and the coupling between the QD and leads is weak. When we apply a more positive top gate voltages to realize stronger coupling, we also observe a similar Coulomb oscillation as a function of $V_{\mathrm{SG}}$. For such top gate conditions, the valley conductance as well as the Coulomb peaks seem to be enhanced. In addition, we observe peculiar plateaulike features as shown in Fig.4(a), where $V_{T G}$ was set to $-266.25 \mathrm{mV}$. This plateau possibly originates from the Kondo effect. The Kondo effect in QDs appears when the coupling between the leads and the QD is strong and the ground state has a net spin (usually $S=1 / 2$ ). Firstly, the width of the plateau is considerably larger than the other Coulomb oscillations (see the peak at $V_{S G}=-1.05 \mathrm{~V}$ ). It also seems to split into two peaks whose widths are comparable to the other peaks when the top gate voltage is slightly changed either to the negative or positive direction, indicating that the original feature consists of two Coulomb oscillation peaks. Secondly, the conductance of the plateau is extremely large, reach- ing $1.7 e^{2} / h$. Furthermore, when we measure the differential conductance we clearly observe a characteristic peak at $V_{D S}=0$, only when $V_{S G}$ is in the plateau region, as shown in Fig. 4(b). The width of the zero bias peak is about $80 \mu \mathrm{V}$ (see inset of Fig. 4(a)). Furthermore, application of the magnetic field perpendicular to the dot also suppresses the plateau-like feature as shown in the region enclosed by the black box in Fig.4(c). In Fig. 4(c), we also can see a pair of Coulomb peaks which shows similar shifts with respect to $B$ at both positive and negative $V_{S G}$ side of the plateau, suggesting that the number of the electrons in the valley is odd and the spin degeneracy in the ground state.

Interestingly, we observe features related to the Kondo effect up to very high magnetic field $(\sim 5 \mathrm{~T})$ in this device. A detailed study of the Kondo effect at high magnetic field will be described elsewhere[9].

\section{Summary}

We have succeed in the fabrication of novel single electron transistors based on a quantum dot device fabricated by selective area growth of metalorganic vapor phase epitaxy in combination with fine gate structures. Clear Coulomb oscillations and Coulomb diamonds are observed at low temperatures. Signatures of Kondo effect are also observed when the coupling between the leads and the quantum dot is made strong. 


\section{References}
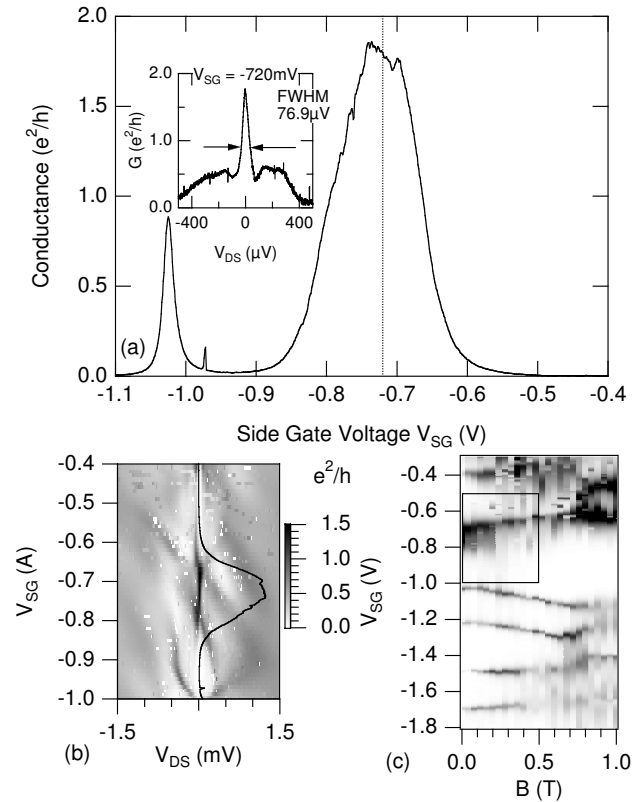

Fig. 4. (a) Coulomb oscillation when the top gate voltage is set to $V_{T G}=-266.25 \mathrm{mV}$. Inset: Differential conductance $d I_{D S} / V_{D S}$ for $V_{S G}=-720 \mathrm{mV}$ (indicated by dotted line). (b) Grey scale plot of $d I_{D S} / V_{D S}$, plotted as a function of both $V_{D S}$ and $V_{S G}$ for $V_{T G}=-266.25 \mathrm{mV}$. (c) Magnatic field dependence of the Coulomb oscillations.

\section{Acknowledgments}

The authors acknowledge Prof. H. Hasegawa, Prof. H. Akera, Prof. M. Eto, and Prof. S. Kasai for fruitful discussions. and Dr. Y. Oda, T. Terasawa, T. Harada, and T. Kusuhara for experimental support. This work is partly financially supported by a Grant-in-Aid for Scientific Research, supported by Ministry of Education, Culture, Sports, Science and Technology, Japan.
[1] K. K. Likharev, IEEE Trans. Magn. 23, 1142 (1987).

[2] S. Tarucha, D.G. Austing, T. Honda, R.J. van der Hage, and L.P. Kouwenhoven, Phys. Rev. Lett. 77, 3613 (1996).

[3] D. Goldharber-Gordon, H. Shtrikman, D. Mahalu, D. Abusch-Magder, U. Meirav, M.A. Kastner, Nature 391, 156 (1998); S.M. Cronenwett, T.H. Oosterkamp, L.P. Kouwenhoven, Science 281, 540 (1998); J. Schmid, J. Weiss, K. Eberl, and K.v. Klitzing, Physica B 256-258, 182 (1998).

[4] K. Kumakura, J. Motohisa, and T. Fukui, Solid State Electron. 42, 1227 (1998); K. Kumakura, J. Motohisa, and T. Fukui, Physica E 2, 809 (1998).

[5] F. Nakajima, K. Kumakura, J. Motohisa, and T. Fukui, Jpn. J. Appl. Phys. 38, 415 (1999).

[6] F. Nakajima, J. Motohisa, and T. Fukui, Appl. Surf. Sci. 162-163, 650 (2000).

[7] F. Nakajima, Y. Ogasawara, J. Motohisa, and T. Fukui, to be published in J. Appl. Phys.

[8] M. Digler, R.J. Haug, K. Eberl and K. von Klitzing, Semicond. Sci. and Technol. 11, 1493 (1996).

[9] W.G. van der Weil et al., to be published. 